\title{
Privacy preserving social network data publication
}

\begin{abstract}
The introduction of online social networks (OSN) has transformed the way people connect and interact with each other as well as share information. OSN have led to a tremendous explosion of network-centric data that could be harvested for better understanding of interesting phenomena such as sociological and behavioural aspects of individuals or groups. As a result, online social network service operators are compelled to publish the social network data for use by third party consumers such as researchers and advertisers. As social network data publication is vulnerable to a wide variety of reidentification and disclosure attacks, developing privacy preserving mechanisms are an active research area. This paper presents a comprehensive survey of the recent developments in social networks data publishing privacy risks, attacks, and privacy-preserving techniques. We survey and present various types of privacy attacks and information exploited by adversaries to perpetrate privacy attacks on anonymized social network data. We present an in-depth survey of the state-of-the-art privacy preserving techniques for social network data publishing, metrics for quantifying the anonymity level provided, and information loss as well as challenges and new research directions. The survey helps readers understand the threats, various privacy preserving mechanisms, and their vulnerabilities to privacy breach attacks in social network data publishing as well as observe common themes and future directions.
\end{abstract}

Keyword: Social network data; Privacy attacks; Anonymized graphs; Privacy preserving; Data privacy 